Original Paper http://ajol.info/index.php/ijbcs http://indexmedicus.afro.who.int

\title{
Synthesis of symetrical near-infrared dyes containing benzo[c,d]indole system
}

\author{
Venance Martial SAY*, Timothée Aboua OKPEKON, Sagne Jacques AKPA and \\ Ané ADJOU
}
Laboratoire de Chimie Organique et de Substances Naturelles (LCOSN), UFR SSMT, Université Félix Houphouët Boigny, 22 BP 582 Abidjan 22, Côte d'Ivoire.
*Corresponding author; E-mail: martialvs@gmail.com; Tel: 0022507052119

\section{ACKNOWLEDGMENTS}

The authors thank the Chemistry Department of Georgia State University for the support on the NMR, MALDI-MS and UV measurements.

\begin{abstract}
Five symmetrical near infrared dyes having different linker between the two heterocycles were synthesized by condensation of $\mathrm{N}$-alkylbenzo[c,d]indolium salts with either squaric acid or imine. All synthesized dyes showed strong absorbance in the near-infrared region of 850-1015 nm and can be used as nucleic acid detection or fluorescent labeling agents for proteins. Three of the dyes $\mathbf{2 1 ,} 22$ and 25 containing functionalized $N$-substituents (ester group) can be further modified or covalently attached to target. The structures of these cyanine dyes where characterized by virtue of NMR, UV, MALDI and Elemental analysis. (C) 2019 International Formulae Group. All rights reserved
\end{abstract}

Keywords: Synthesis, cyanine, Polymethine dyes, Near-IR dyes, Absorption, Chromophore.

\section{INTRODUCTION}

Cyanine dyes are a large class of synthetic polymethine dyes with a wide variety of colors that can show absorptions from the ultraviolet to the infrared region. They have two terminal aza-heterocycles connected via an electron deficient polymethine bridge that allows for a push/pull system between the two heterocycles. The delocalization of electrons across this bridge causes them to exhibit long wavelength absorptions. Due to the diversity in function associated with this class of chromophore, an extensive number of cyanine dyes have been synthesized and developed for numerous applications including optical recording using a Ga-Al-As diode laser (Volkva et al., 2007; Rauch et al., 2009; Zhang et al., 2015; Liu et al., 2016) and fluorescent probes for biomolecular labeling and imaging (Bouteiller $\mathrm{C}$ et al., 2007; Pisoni et al., 2014; Hyun et al., 2015; Njiojob et al., 2015). Also, the heterocycles themselves can be altered which allow chemists to create dyes that possess ideal photophysical properties, such as high molar extinction coefficients $\left(>10^{5} \mathrm{M}^{-1} \mathrm{~cm}^{-1}\right)$ and narrow absorption bands. The most common heterocyclic end units in cyanine and other polymethine dyes are cationic indolium and benzo-fused indolium moieties. The 
longer wavelength region (>800 $\mathrm{nm})$ is characterized by greatly reduced background fluorescence of any complex matrix. In addition, since Raman scattering shows $1 / \lambda^{4}$ dependence, the background is further decreased in the NIR region.

In this study, we describe the preparation of five symmetrical near infrared dyes having different linker between the two heterocycles which can be used as nucleic acids detection or fluorescent labeling agents for proteins by condensation of $\mathrm{N}$ alkylbenzo[c,d]indolium salts with either squaric acid or imine.

\section{MATERIALS AND METHODS}

\section{General}

Melting points were determined using a Kofler bench and are uncorrected. Purifications by column chromatography were carried out on Kieselgel 60 (230-400 mesh, Merck). ${ }^{1} \mathrm{H}$ and ${ }^{13} \mathrm{C}$ NMR spectra were measured on a $300 \mathrm{MHz}$ Bruker Avanced apparatus with tetramethylsilane (TMS) as internal standard. The electronic absorption spectra were recorded on a Shimadzu UV3101 or 2401 PC UV-VIS-NIR scanning spectrophotometer.

\section{Experimental}

Synthesis of 1-alkylbenz[c,d]indole-2(1H)one 2 and 3

To a solution of benz[c,d]indol-2(1H)one $(1.5 \mathrm{~g}, 8.9 \mathrm{mmol})$ in $15 \mathrm{~mL}$ of $\mathrm{N}$-methyl2-pyrrolidinone, we added $40 \%$ sodium hydroxide $(0.7 \mathrm{ml}, 22.3 \mathrm{mmol})$. Then, slowly, acylating agent was added dropwise. The reaction mixture was heated at $50{ }^{\circ} \mathrm{C}$ for $1 \mathrm{~h}$, after which time no starting material was present as shown by thin layer chromatography (TLC). After cooling at room temperature, water $(10 \mathrm{ml})$ was added, extracted with dichloromethane and washed with brine. The extract was dried over anhydrous magnesium sulfate, filtered and concentrated. The crude product was purified by flash chromatography on silica gel using $\mathrm{AcOEt} / \mathrm{C}_{6} \mathrm{H}_{14}(1: 10)$ as eluent.
Synthesis of 1-alkylbenz[c,d]indole $-2(1 \mathrm{H})-$ thione 4 and 5

A mixture of amide 2 (200 $\mathrm{mg}, 0.59$ $\mathrm{mmol})$ or 3 (200 mg, $1.01 \mathrm{mmol})$ and diphosphorus pentasulphide (365 mg, 1.64 mmol) in pyridine $(8 \mathrm{ml})$ was refluxed for $2 \mathrm{~h}$. The cooled reaction mixture was acidified with concentrated $\mathrm{HCl}$ and the red product which precipitated by cooling was filtered and dried.

Synthesis of 1-ethyl-2thiomethylbenz[c,d]indolium iodide 6

Methyl iodide $(0.6 \mathrm{~mL}, 9.4 \mathrm{mmol})$ was added to 1-ethyllbenz[c,d]indole-2 $(1 \mathrm{H})$-thione $(0.94 \mathrm{mmol})$. The mixture was stirred at room temperature for $14 \mathrm{~h}$. Product was filtered off, washed with acetone and dried. It was used for the next stage without further purification.

Synthesis of 1-ethylphenylsulfone-2thiomethylbenz[c,d]indolium sulfate 7

Dimethylsulfate $(3.2 \mathrm{ml}, 25.2 \mathrm{mmol})$ was added to 1-ethyllbenz[c,d]indole $-2(1 \mathrm{H})$ thione $(2.4 \mathrm{~g}, 5.04 \mathrm{mmol})$ and toluene $(8 \mathrm{ml})$. The mixture was heated at $130{ }^{\circ} \mathrm{C}$ for $2 \mathrm{~h}$. No solid was formed. However, a sticky oil was formed, the solvent was removed and dried, and used for the next step.

Synthesis of 1-alkyl-2-(2,2-dimethyl-4,6dioxo-1,3-dioxan-5-ylidene)-1(H)benz[c,d]indole 8 and 9

\section{2,2-Dimethyl-1,3-dioxan-4,6-dione}

$(1.4 \mathrm{mmol})$ and triethylamine $(1.4 \mathrm{mmol})$ were added to a solution of 1-ethyl-2thiomethylbenz[c,d]indolium iodide $(0.45$ $\mathrm{mmol})$ in absolute ethanol $(6 \mathrm{ml})$. The reaction mixture was refluxed for $2 \mathrm{~h}$ and, after cooling, water was added to precipitate the desired product which was filtered off and washed with water and dried.

\section{Synthesis of 1-alkyl-2-} methylbenzo[c,d]indolium iodide 10 and 11

A solution of 8 or $9(2.7 \mathrm{mmol})$ and acetic acid $(5 \mathrm{ml})$ was refluxed and concentrated $\mathrm{HCl}$ was added dropwise to the refluxed solution until the color disappeared. Saturated KI solution $(12 \mathrm{ml})$ was added to the cooled reaction mixture to precipitate the product which was then filtered off and dried. 


\section{Synthesis of squaraine dyes 12 and 13}

A mixture of a quaternary salt $\mathbf{1 0}(300$ $\mathrm{mg}, 0.65 \mathrm{mmol})$ and squaric acid $(37 \mathrm{mg}, 0.32$ mmol) was heated under reflux in $6 \mathrm{~mL}$ of toluene/butanol (1:1) for $2 \mathrm{~h}$. After cooling, product was filtered off and washed with ethyl ether. Pure product was obtained by purification on chromatotron (12) or on silica gel column chromatography, both using $\mathrm{CH}_{2} \mathrm{Cl}_{2} / \mathrm{MeOH}$ (9:1) as an eluent, followed by recrystallization from methanol/hexane (13) (Scheme 2).

\section{Synthesis of benz[c,d]indole -2(1H)-thione} (14)

A mixture of amide $(17.73 \mathrm{mmol})$ and diphosphorus pentasulphide $(17.73 \mathrm{mmol})$ in pyridine $(30 \mathrm{ml})$ was refluxed for $2 \mathrm{~h}$. The resulting solution was decanted from a little tar into warm water, and the mixture was warmed on the steam-bath. After cooling, the oil gave a solid, which was crystallized from ethanol to yield the desired product 14.

\section{Synthesis of thiomethylbenz[c,d]indolium iodide (15)}

Methyl iodide (9.4 mmol, $0.6 \mathrm{ml}$ ) was added to benz[c,d]indole $-2(1 \mathrm{H})$-thione $(0.94$ $\mathrm{mmol})$. The mixture was stirred at room temperature for $14 \mathrm{~h}$. Product was filtered off, washed with acetone and dried. It was used for the next stage without further purification.

\section{Synthesis 2-(2,2-Dimethyl-4,6-dioxane-5-} yliden)(1H)benz[c,d]indole (16)

2,2-Dimethyl-1,3-dioxan-4,6-dione

$(3.1 \mathrm{mmol})$ and triethylamine $(3.1 \mathrm{mmol})$ were added to a solution of thiomethylbenz[c,d]indolium iodide $(0.77$ mmol) in absolute ethanol $(15 \mathrm{ml})$. The reaction mixture was refluxed for $2 \mathrm{~h}$ and, after cooling, water was added to precipitate the desired product which was filtered off and washed with water and dried.

Synthesis of ethyl 6-(2-(2,2-dimethyl-4,6dioxo-1.3dioxan-5-ylidene)benzo[c,d]indol1(2H)-yl)hexanoate (17)

A mixture of 16 ( $3 \mathrm{~g}, 10.40 \mathrm{mmol})$, ethyl-6-bromohexanoate $(5.6 \mathrm{~g}, 31.30 \mathrm{mmol})$ and $\mathrm{K}_{2} \mathrm{CO}_{3}(4.4 \mathrm{~g}, 31.30 \mathrm{mmol})$ was heated at
90-100 ${ }^{\circ} \mathrm{C}$ in DMF (40 ml) for $15 \mathrm{~h}$. After filtration, the filtrate was condensed and the crude product $\mathbf{1 7}$ was purified by flash chromatography on silica gel $\left(\mathrm{AcOEt} / \mathrm{C}_{6} \mathrm{H}_{14}\right.$, 3:7) (Scheme 1).

Synthesis of 1-(5-carboxypentyl)-2methylbenzo[c,d]indolium inner salt (18)

A solution of $\mathbf{1 7}(3 \mathrm{~g})$ and acetic acid was refluxed and concentrated $\mathrm{HCl}$ was added dropwise to the refluxed solution until the color disappeared. Saturated KI solution (30 $\mathrm{mL}$ ) was added to the cooled reaction mixture to precipitate the product which was then filtered off and dried (Scheme 1).

Synthesis of Cyanine Dyes of 21, 22 and 25

A mixture of salt 18 (2.36 mmol), animine $(1.15 \mathrm{mmol})$, anhydrous sodium acetate $(4 \mathrm{mmol})$ and $25 \mathrm{ml}$ of acetic anhydride was heated at $80{ }^{\circ} \mathrm{C}$ for $3 \mathrm{~h}$ (Scheme 4). The crude mixture was condensed to get a solid; this solid was then dissolved in $20 \mathrm{ml}$ of DMF (some of the inorganic compounds did not dissolve) and filtered. The filtrate was condensed, dried and used for the next step. Thus, to this dried mixture of the crude, dry $\mathrm{K}_{2} \mathrm{CO}_{3}(9.8 \mathrm{mmol})$ and EtI $(11 \mathrm{mmol})$ were added and stirred at room temperature in anhydrous DMF (30 ml) for $20 \mathrm{~h}$. TLC analysis showed no presence of starting material. After filtration of the inorganic products, the filtrate was condensed to get a "gummy" crude product. Products were purified respectively by column chromatography then chromatotron using $\mathrm{CH}_{2} \mathrm{Cl}_{2} / \mathrm{MeOH}(9: 1)$ as eluent.

Synthesis of Cyanine dye 25

A mixture of the crude product of $\mathbf{2 4}$ ( 2 $\mathrm{g}, 2.45 \mathrm{mmol})$, dry $\mathrm{K}_{2} \mathrm{CO}_{3}(1.6 \mathrm{~g}, 11.6 \mathrm{mmol})$ and EtI ( $3.6 \mathrm{ml}, 13 \mathrm{mmol})$ was stirred at room temperature in anhydrous DMF (30 mL) for $20 \mathrm{~h}$. TLC analysis showed no presence of starting material 24. After filtration, the filtrate was condensed to get a "gummy" crude product. Product 25 was purified by using successively column chromatography then chromatotron. 

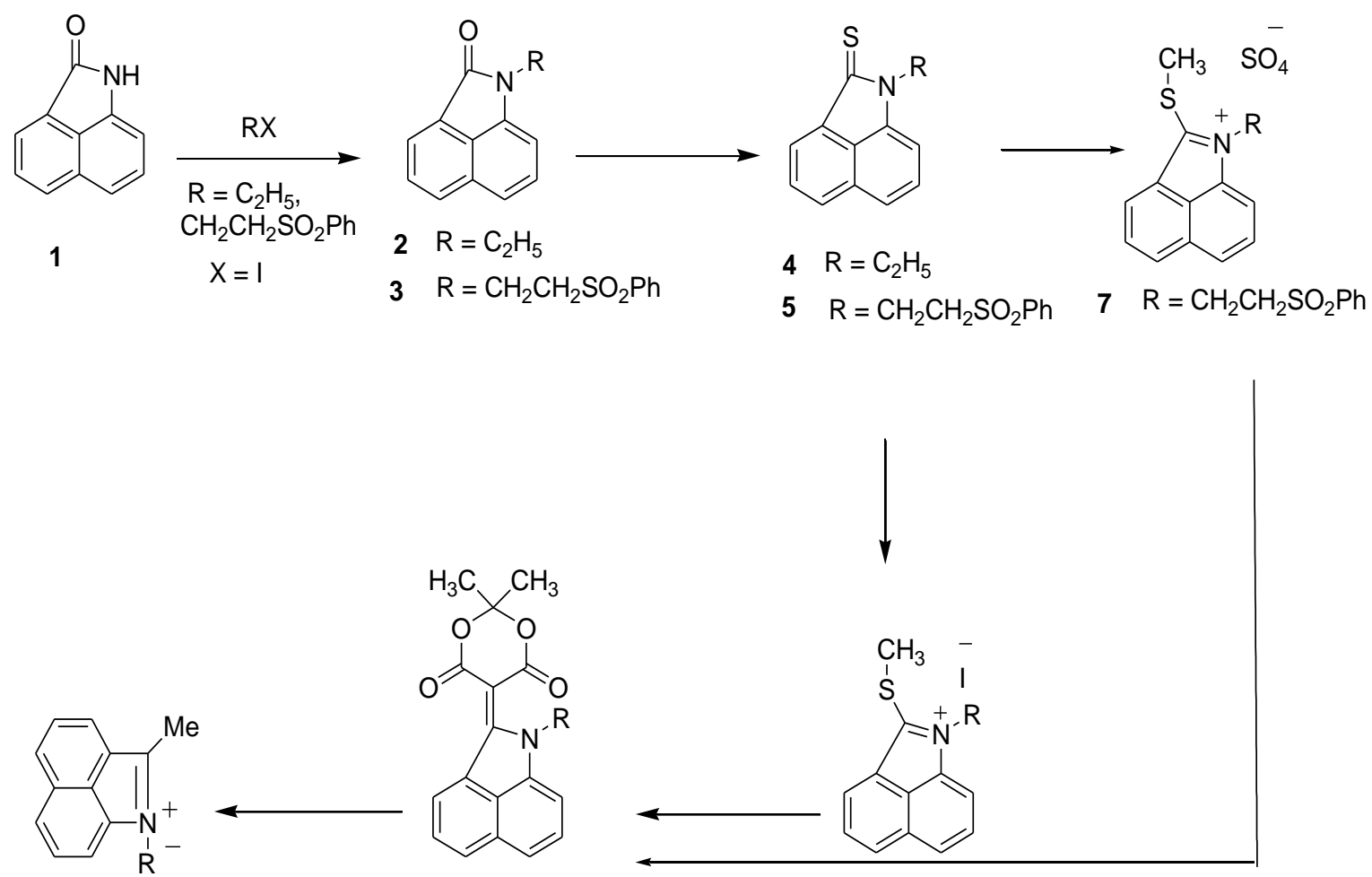

$10 \mathrm{R}=\mathrm{C}_{2} \mathrm{H}_{5}$

$11 \mathrm{R}=\mathrm{CH}_{2} \mathrm{CH}_{2} \mathrm{SO}_{2} \mathrm{Ph}$

$\begin{array}{ll}8 & \mathrm{R}=\mathrm{C}_{2} \mathrm{H}_{5} \\ 9 & \mathrm{R}=\mathrm{CH}_{2} \mathrm{CH}_{2} \mathrm{SO}_{2} \mathrm{Ph}\end{array}$

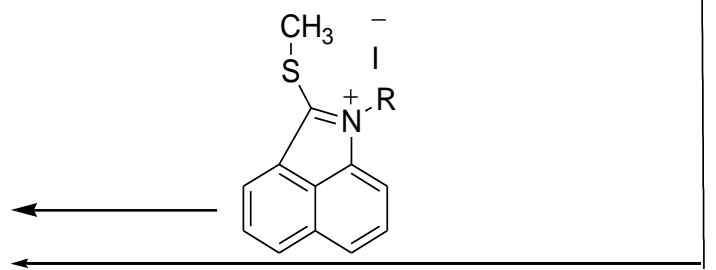

$6 \mathrm{R}=\mathrm{C}_{2} \mathrm{H}_{5}$

Scheme 1: Synthesis of the $N$-alkylbenzo[c,d]indolium salts 10 and 11 .

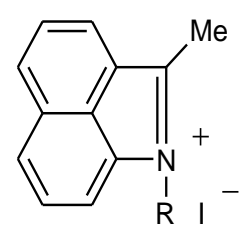

10: $\mathrm{R}=\mathrm{Et}$

11: $\mathrm{R}=\mathrm{CH}_{2} \mathrm{CH}_{2} \mathrm{SO}_{2} \mathrm{Ph}$

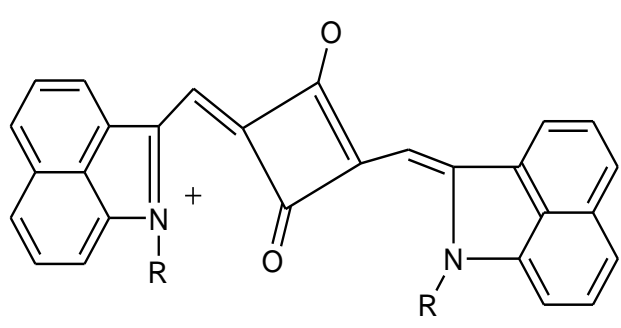

12: $\mathrm{R}=\mathrm{Et}$

13: $\mathrm{R}=\mathrm{CH}_{2} \mathrm{CH}_{2} \mathrm{SO}_{2} \mathrm{Ph}$

Scheme 2: Synthesis of squaraine dyes 12 and 13. 
<smiles>O=C1Nc2cccc3cccc1c23</smiles>

1

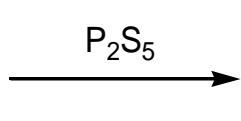<smiles>CCCCCn1c(=C2C(=O)OC(C)(C)OC2=O)c2cccc3cccc1c32</smiles>

14

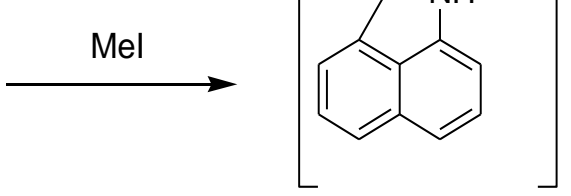

15

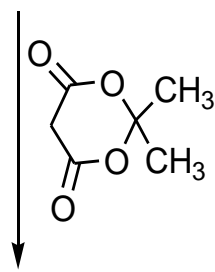

$\mathrm{BrC}_{5} \mathrm{H}_{10} \mathrm{CO}_{2} \mathrm{C}_{2} \mathrm{H}_{5}$<smiles>CC1(C)OC(=O)C(=c2[nH]c3cccc4cccc2c43)C(=O)O1</smiles>

16<smiles></smiles>

18

Scheme 3: Synthesis of 1-(5-carboxypentyl)-2-methylbenzo[c,d]indolium inner salt. 


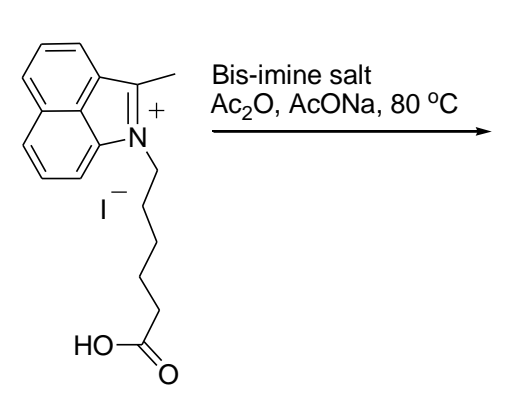

18

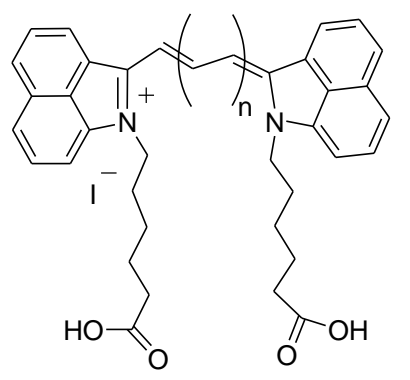

19: $n=2$
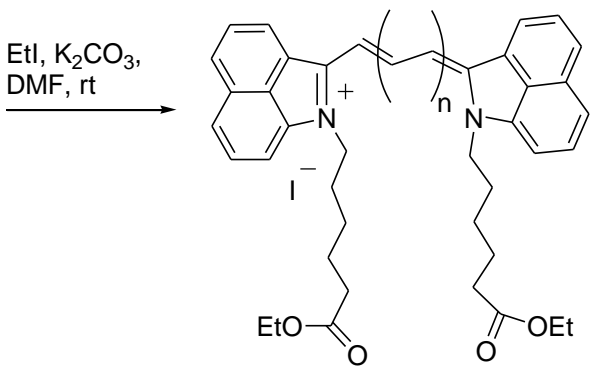

21: $n=2$

22: $\mathrm{n}=3$

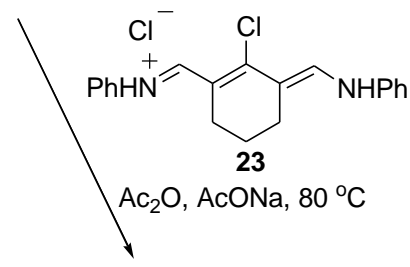

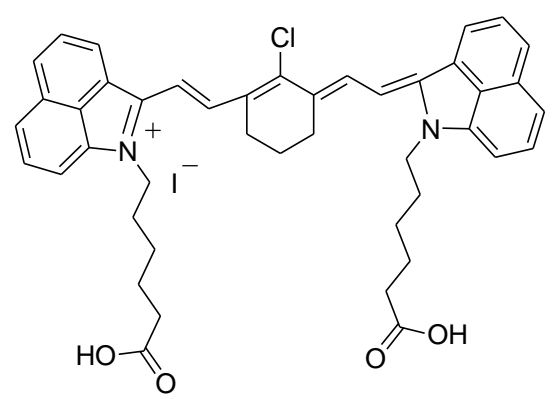

24
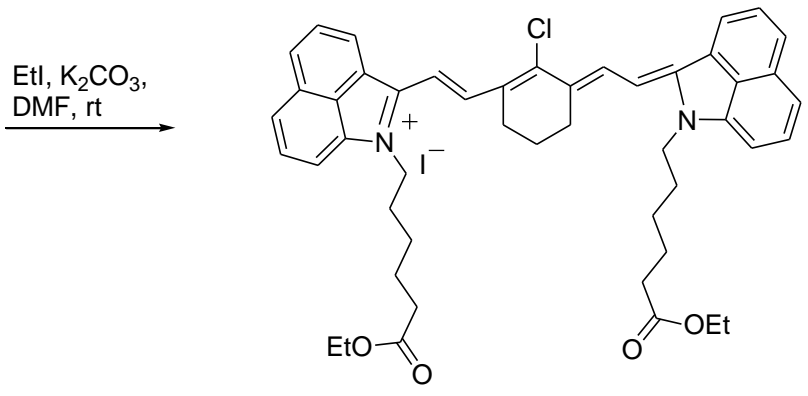

25

Scheme 4: Synthesis of ester dyes.

\section{RESULTS}

The physical and spectrometric data of all synthesized compounds are reported below.

\section{1-Ethyllbenz $[\mathrm{c}, \mathrm{d}]$ indole-2(1H)-one (2)}

Yield 89\%; M.p: 68-69 ${ }^{\circ} \mathrm{C} ;{ }^{1} \mathrm{H}-\mathrm{NMR}$ $\left(\right.$ DMSO- $\left.d_{6}\right) \delta 8.18(\mathrm{~d}, J=8 \mathrm{~Hz}, 1 \mathrm{H},-\mathrm{ArH})$, $8.05(\mathrm{~d}, J=8.0 \mathrm{~Hz}, 1 \mathrm{H},-\mathrm{ArH}), 7.80(\mathrm{~m}, 1 \mathrm{H},-$ ArH), $7.64(\mathrm{~d}, 1 \mathrm{H},, J=7.5 \mathrm{~Hz},-\mathrm{ArH}), 7.54$ (m, 1H, -ArH), 7.20 (d, $J=7.5 \mathrm{~Hz}, 1 \mathrm{H},-\mathrm{ArH})$, $4.71\left(\mathrm{q}, J=7.0 \mathrm{~Hz}, 2 \mathrm{H},-\mathrm{CH}_{2} \mathrm{CH}_{3}\right), 1.28(\mathrm{t}, J=$ $7.0 \mathrm{~Hz}, 3 \mathrm{H},-\mathrm{CH}_{2} \mathrm{CH}_{3}$ ).

\section{1-(2-(Phenylsulfonyl)ethyl)benz $[c, d]$ indole- 2(1H)-one (3)}

Yield 50\%; M.p: $155-156{ }^{\circ} \mathrm{C} ;{ }^{1} \mathrm{H}-\mathrm{NMR}$ $\left(\right.$ DMSO- $\left.d_{6}\right) \delta, 8.14(\mathrm{t}, J=8.5 \mathrm{~Hz}, 1 \mathrm{H},-\mathrm{ArH})$, $7.95(\mathrm{t}, J=8 \mathrm{~Hz}, 1 \mathrm{H},-\mathrm{ArH}), 7.76(\mathrm{~m}, 3 \mathrm{H}$, -
$\operatorname{ArH}), 7.61(\mathrm{t}, J=8.5 \mathrm{~Hz}, 1 \mathrm{H},-\mathrm{ArH}), 7.51(\mathrm{~m}$, $2 \mathrm{H},,-\operatorname{ArH}), 7.41(\mathrm{~m}, 2 \mathrm{H},-\mathrm{ArH}), 7.11(\mathrm{t}, J=$ $8.0 \mathrm{~Hz}, 1 \mathrm{H},-\mathrm{ArH}), 4.22(\mathrm{t}, J=7.0 \mathrm{~Hz}, 2 \mathrm{H},-$ $\mathrm{CH}_{2} \mathrm{CH}_{2} \mathrm{SO}_{2} \mathrm{Ph}$ ), $3.88(\mathrm{t}, J=7.0 \mathrm{~Hz}, 2 \mathrm{H}$, $\mathrm{CH}_{2} \mathrm{CH}_{2} \mathrm{SO}_{2} \mathrm{Ph}$ ).

\section{1-Ethyllbenz[c,d]indole-2(1H)-thione (4)} Yield 89\%; M.p: $126-128{ }^{\circ} \mathrm{C} ;{ }^{1} \mathrm{H}-\mathrm{NMR}$ $\left(\mathrm{DMSO}-d_{6}\right) \delta 8.22(\mathrm{~d}, J=8.0 \mathrm{~Hz}, 1 \mathrm{H},-\mathrm{ArH})$ $8.13(\mathrm{~d},, J=8.0 \mathrm{~Hz}, 1 \mathrm{H},-\mathrm{ArH}), 7.77(\mathrm{~m}, 2 \mathrm{H}$, -ArH), $7.61(\mathrm{t}, J=7.0 \mathrm{~Hz}, 1 \mathrm{H},-\mathrm{ArH7} .40$ (t, $J$ $=7.0 \mathrm{~Hz}, 1 \mathrm{H},-\mathrm{ArH}),), 4.41(\mathrm{q}, J=7 \mathrm{~Hz}, 2 \mathrm{H}$, $\left.-\mathrm{CH}_{2} \mathrm{CH}_{3}\right), 1.34\left(\mathrm{t}, J=7.0 \mathrm{~Hz}, 3 \mathrm{H},-\mathrm{CH}_{2} \mathrm{CH}_{3}\right.$ ).

\section{1-(2-(Phenylsulfonyl) ethyl)benz[c,d]indole-} 2(1H)-thione (5)

Yield 90\%; M.p: $154-155^{\circ} \mathrm{C}$; ${ }^{1} \mathrm{H}-\mathrm{NMR}$ $\left(\mathrm{DMSO}-d_{6}\right) \delta 8.20(\mathrm{~d}, J=8.0 \mathrm{~Hz}, 1 \mathrm{H},-\mathrm{ArH})$, $8.03(\mathrm{~d}, J=7.5 \mathrm{~Hz}, 1 \mathrm{H},-\mathrm{ArH}), 7.75(\mathrm{~m}, 4 \mathrm{H},-$ 
ArH), 7.57 (m, 2H, $-\mathrm{ArH}), 7.41(\mathrm{t}, J=8.0 \mathrm{~Hz}$, $2 \mathrm{H},-\mathrm{ArH}), 7.32(\mathrm{t}, J=7.5 \mathrm{~Hz}, 1 \mathrm{H},-\mathrm{ArH})$, $4.63\left(\mathrm{t},, J=7.0 \mathrm{~Hz}, 2 \mathrm{H},-\mathrm{CH}_{2} \mathrm{CH}_{2} \mathrm{SO}_{2} \mathrm{Ph}\right.$ ), $3.97\left(\mathrm{t}, J=7.0 \mathrm{~Hz}, 2 \mathrm{H},-\mathrm{CH}_{2} \mathrm{CH}_{2} \mathrm{SO}_{2} \mathrm{Ph}\right)$.

\section{1-Ethyl-2-(2,2-dimethyl-4,6-dioxo-1,3-} dioxan-5-ylidene)-1(H)-benz[c,d]indole (8)

Yield 65\%; M.p: $183-184{ }^{\circ} \mathrm{C} ;{ }^{1} \mathrm{H}-\mathrm{NMR}$ $\left(\right.$ DMSO- $\left.d_{6}\right) \delta 8.90(\mathrm{~d}, \mathrm{~J}=8.0 \mathrm{~Hz}, 1 \mathrm{H},-\mathrm{ArH})$ $8.40(\mathrm{~d}, \mathrm{~J}=8.0 \mathrm{~Hz}, 1 \mathrm{H},-\mathrm{ArH}), 8.01(\mathrm{~m}, 1 \mathrm{H},-$ $\mathrm{ArH}), 7.91(\mathrm{~m}, 2 \mathrm{H},-\mathrm{ArH}), 7.78(\mathrm{~m}, 1 \mathrm{H},-$ $\mathrm{ArH}), 4.39$ (q, $J=7.0 \mathrm{~Hz}, 2 \mathrm{H},-\mathrm{CH}_{2} \mathrm{CH}_{3}$ ), $1.70\left(\mathrm{~s}, 6 \mathrm{H},-\mathrm{O} 2\left(\mathrm{CH}_{3}\right)_{2}\right), 1.46(\mathrm{t}, J=7.0 \mathrm{~Hz}$, $\left.3 \mathrm{H},-\mathrm{CH}_{2} \mathrm{CH}_{3}\right)$.

\section{1-(2-(Phenylsulfonyl)ethyl-2-(2,2-dimethyl-}

\section{4,6-dioxo-1,3-dioxan-5-ylidene)-1(H)-} benz $[\mathbf{c}, \mathbf{d}]$ indole $(9)$

Yield 65\%; M.p: $180-181{ }^{\circ} \mathrm{C} ;{ }^{1} \mathrm{H}$ NMR (DMSO- $\left.d_{6}\right) \delta 8.90(\mathrm{~d}, J=7.5 \mathrm{~Hz}, 1 \mathrm{H},-\mathrm{ArH})$, $8.33(\mathrm{~d}, J=8.0 \mathrm{~Hz}, 1 \mathrm{H},-\operatorname{ArH}), 7.95(\mathrm{~d}, J=$ $8.0 \mathrm{~Hz}, 1 \mathrm{H},-\mathrm{ArH}), 7.90$ (d, $J=7.5 \mathrm{~Hz}, 1 \mathrm{H}$, ArH), $7.83(\mathrm{~d}, J=7.5 \mathrm{~Hz}, 1 \mathrm{H},-\mathrm{ArH}), 7.70(\mathrm{~d}$, $J=7.5 \mathrm{~Hz}, 1 \mathrm{H},-\mathrm{ArH}), 7.63(\mathrm{~m}, 2 \mathrm{H},-\mathrm{ArH})$, 7.33 (m, 1H, -ArH), 7.23 (m, 2H, -ArH), 4.22 $\left(\mathrm{t}, J=7.0 \mathrm{~Hz}, 2 \mathrm{H},-\mathrm{CH}_{2} \mathrm{CH}_{2} \mathrm{SO}_{2} \mathrm{Ph}\right), 3.88(\mathrm{t}, J$ $\left.=7 \mathrm{~Hz}, 2 \mathrm{H}, \mathrm{CH}_{2} \mathrm{CH}_{2} \mathrm{SO}_{2} \mathrm{Ph}\right), 1.72(\mathrm{~s}, 6 \mathrm{H}$, $\left.\mathrm{O} 2\left(\mathrm{CH}_{3}\right)_{2}\right)$.

\section{1-Ethyl-2-methylbenzo[c,d]indolium iodide} (10)

Yield 76\%, M.p: $242-244{ }^{\circ} \mathrm{C} ;{ }^{1} \mathrm{H}-$ NMR (DMSO- $\left.d_{6}\right) \delta 8.79(\mathrm{~d}, J=8.0 \mathrm{~Hz}, 1 \mathrm{H}$, $\mathrm{ArH}), 8.69(\mathrm{~d}, J=8.0 \mathrm{~Hz}, 1 \mathrm{H},-\mathrm{ArH}), 8.54(\mathrm{~d}$, $J=8.0 \mathrm{~Hz}, 1 \mathrm{H},-\mathrm{ArH}), 8.45(\mathrm{~d}, J=8.0 \mathrm{~Hz}$, $1 \mathrm{H},-\mathrm{ArH}), 8.19(\mathrm{~m}, 1 \mathrm{H},-\mathrm{ArH}), 8.0(\mathrm{~m}, 1 \mathrm{H},-$ $\mathrm{ArH}), 4.71\left(\mathrm{q}, J=7.0 \mathrm{~Hz}, 2 \mathrm{H},-\mathrm{CH}_{2} \mathrm{CH}_{3}\right), 4.46$ $\left(\mathrm{s}, 3 \mathrm{H},-\mathrm{CH}_{3}\right), 1.56\left(\mathrm{t}, J=7 \mathrm{~Hz}, 3 \mathrm{H},-\mathrm{CH}_{2} \mathrm{CH}_{3}\right)$.

\section{1-(2-(Phenylsulfonyl)ethyl-2-}

methylbenzo[c,d]indolium iodide (11)

Yield 40\%; M.p: $280-282{ }^{\circ} \mathrm{C} ;{ }^{1} \mathrm{H}-$ NMR (DMSO- $\left.d_{6}\right) \delta 8.42(\mathrm{~d}, J=8.0 \mathrm{~Hz}, 1 \mathrm{H}$, $\mathrm{ArH}), 8.00(\mathrm{~d}, J=7.5 \mathrm{~Hz}, 1 \mathrm{H},-\mathrm{ArH}), 7.75(\mathrm{~m}$, $4 \mathrm{H},-\mathrm{ArH}), 7.57(\mathrm{~m}, 2 \mathrm{H},-\mathrm{ArH}), 7.31(\mathrm{t}, J=$ $8.0 \mathrm{~Hz}, 2 \mathrm{H},-\mathrm{ArH}), 7.22(\mathrm{t}, J=7.5 \mathrm{~Hz}, 1 \mathrm{H}$, $\mathrm{ArH}), \quad 4.62(\mathrm{t}, \quad J=7.0 \mathrm{~Hz}, \quad 2 \mathrm{H}, \quad-$ $\mathrm{CH}_{2} \mathrm{CH}_{2} \mathrm{SO}_{2} \mathrm{Ph}$ ), 4.35 (s, $\left.3 \mathrm{H},-\mathrm{CH}_{3}\right), 3.90$ (t, $J$ $=7.0 \mathrm{~Hz}, 2 \mathrm{H},-\mathrm{CH}_{2} \mathrm{CH}_{2} \mathrm{SO}_{2} \mathrm{Ph}$ ).

\section{Squraine dye 12}

Yield $13 \% ; \lambda_{\max }\left(\mathrm{CH}_{2} \mathrm{Cl}_{2}\right)=880 \mathrm{~nm}$; M.p: > $300{ }^{\circ} \mathrm{C} ;{ }^{1} \mathrm{H}-\mathrm{NMR}$ (DMSO-d $\left.d_{6}\right) \quad \delta 9.20$ (s, 2H, -ArH), 7.94 (d, $J=7.0 \mathrm{~Hz}, 2 \mathrm{H},-\mathrm{ArH})$, 7.85 (m, 2H, -ArH), 7.51 (m, 4H, -ArH), 7.05 (d, $J=7.0 \mathrm{~Hz}, 2 \mathrm{H},-\mathrm{ArH}), 6.36$ (s, $2 \mathrm{H},(-$ $\mathrm{CH}=) 2), 4.25 \quad(\mathrm{q}, \quad J=6.5 \mathrm{~Hz}, 4 \mathrm{H}, \quad(-$ $\left.\left.\mathrm{CH}_{2} \mathrm{CH}_{3}\right) 2\right), 1.28$ (t, $J=6.5 \mathrm{~Hz}, 6 \mathrm{H}$, ($\left.\mathrm{CH}_{2} \mathrm{CH}_{3}\right) 2$ ); Anal. Calcd for $\mathrm{C}_{32} \mathrm{H}_{24} \mathrm{~N}_{2} \mathrm{O}_{2} \cdot 1 / 4$ $\mathrm{H}_{2} \mathrm{O}: \mathrm{C}, 81.24 ; \mathrm{H}, 5.17 ; \mathrm{N}, 5.91$. Found: C, 81.10; H, 5.13; N, 5.93 .

\section{Squaraine dye 13}

Yield 11\%; $\lambda_{\max }\left(\mathrm{CH}_{2} \mathrm{Cl}_{2}\right)=876 \mathrm{~nm}$; M.p: > $300{ }^{\circ} \mathrm{C} ;{ }^{1} \mathrm{H}-\mathrm{NMR}$ (DMSO- $d_{6}$ ) $\delta 8.90$ (s, 2H, ArH), 7.38-7.83 (m, 20H, ArH), 6.25 (s, 2H, (-CH=)2), $4.84(\mathrm{t}, J=7.0 \mathrm{~Hz}, 4 \mathrm{H},(-$ $\left.\left.\mathrm{CH}_{2} \mathrm{CH}_{2} \mathrm{SO}_{2} \mathrm{Ph}\right) 2\right), 4.00$ (t, $J=7.0 \mathrm{~Hz}, 4 \mathrm{H}$, ($\left.\mathrm{CH}_{2} \mathrm{CH}_{2} \mathrm{SO}_{2} \mathrm{Ph}\right) 2$ ); Anal. Calcd for $\mathrm{C}_{44} \mathrm{H}_{32} \mathrm{~N}_{2} \mathrm{O}_{6} \mathrm{~S}_{2} \cdot 2 \mathrm{H}_{2} \mathrm{O}: \mathrm{C}, 67.36 ; \mathrm{H}, 4.58 ; \mathrm{N}$, 3.57. Found: C, 67.14; H, 4.50; N, 4.12.

\section{Benz $[c, d]$ indole -2(1H)-thione (14)}

Yield $85 \%$, M.p: $156-157{ }^{\circ} \mathrm{C}$; ${ }^{1} \mathrm{H}$ NMR ( $300 \mathrm{MHz}$, DMSO- $\left.d_{6}\right) \delta 8.27(\mathrm{~d}, \mathrm{~J}=8 \mathrm{~Hz}, 1$ $\mathrm{H}, \mathrm{ArH}), 8.13(\mathrm{~d}, \mathrm{~J}=8 \mathrm{~Hz}, 1 \mathrm{H}, \mathrm{ArH}), 7.82(\mathrm{t}$, $\mathrm{J}=8 \mathrm{~Hz}, 1 \mathrm{H}, \operatorname{ArH}), 7.75(\mathrm{~d}, \mathrm{~J}=7.5 \mathrm{~Hz}, 1 \mathrm{H}$, $\operatorname{ArH}), 7.51(\mathrm{t}, \mathrm{J}=7 \mathrm{~Hz}, 1 \mathrm{H}, \mathrm{ArH}), 7.2(\mathrm{~d}, \mathrm{~J}=$ $7.5 \mathrm{~Hz}, 1 \mathrm{H}, \mathrm{ArH}) ;{ }^{13} \mathrm{C}$ NMR $(100 \mathrm{MHz}$, DMSO- $\left.d_{6}\right) \quad \delta \quad 108,122.15,125.50,125.66$, $129.80, \quad 129.93, \quad 130.22, \quad 132.23, \quad 134.62$, 140.71, 191.38 .

\section{2-(2,2-Dimethyl-4,6-dioxane-5-}

yliden)(1H)benz $[\mathbf{c}, d]$ indole (16)

Yield 90\%, M.p: $223-224{ }^{\circ} \mathrm{C} ;{ }^{1} \mathrm{H}$ NMR ( $300 \mathrm{MHz}$, DMSO- $\left.d_{6}\right) \delta 9.40(\mathrm{~d}, \mathrm{~J}=8$ $\mathrm{Hz}, 1 \mathrm{H}, \operatorname{ArH}), 8.38$ (d, J = $8 \mathrm{~Hz}, 1 \mathrm{H}, \mathrm{ArH})$, $7.95(\mathrm{~d}, \mathrm{~J}=8 \mathrm{~Hz}, 1 \mathrm{H}, \operatorname{ArH}), 7.85(\mathrm{~m}, 1 \mathrm{H}$, ArH), 7.69 (d, J = $8 \mathrm{~Hz}, 1 \mathrm{H}, \mathrm{ArH}), 7.66$ (m, 1 $\mathrm{H}, \mathrm{ArH}), 1.71\left(\mathrm{~s}, 6 \mathrm{H},-\mathrm{O}_{2}\left(\mathrm{CH}_{3}\right)_{2}\right)$.

\section{Ethyl6-(2-(2,2-dimethyl-4,6-dioxo-}

1.3dioxan-5-ylidene)benzo[c,d]indol-1(2H)yl) hexanoate (17)

Yield 80\%, M.p: $174-175{ }^{\circ} \mathrm{C} ;{ }^{1} \mathrm{H}$ NMR ( $\left.300 \mathrm{MHz}, \mathrm{DMSO}-d_{6}\right) \delta 9.00(\mathrm{~d}, \mathrm{~J}=8.0 \mathrm{~Hz}$, $1 \mathrm{H},-\operatorname{ArH}), 8.17$ ( d, J = $8 \mathrm{~Hz}, 1 \mathrm{H},-\operatorname{ArH})$, $7.82(\mathrm{~m}, 2 \mathrm{H},-\mathrm{ArH}), 7.61(\mathrm{t}, \mathrm{J}=8.0 \mathrm{~Hz}, 1 \mathrm{H}$, 
-ArH), $7.51(\mathrm{~d}, \mathrm{~J}=8 \mathrm{~Hz}, 1 \mathrm{H},-\mathrm{ArH}), 4.39(\mathrm{t}, \mathrm{J}$ $=7.0 \mathrm{~Hz}, 2 \mathrm{H},-\mathrm{NC}_{5} \mathrm{H}_{10} \mathrm{CO}_{2}$-),4.12 (q, J = 7.0 $\left.\mathrm{Hz}, 2 \mathrm{H},-\mathrm{CH}_{2} \mathrm{CH}_{3}\right), 2.31(\mathrm{t}, \mathrm{J}=7.0 \mathrm{~Hz}, 2 \mathrm{H}$, $\left.\mathrm{NC}_{5} \mathrm{H}_{10} \mathrm{CO}_{2^{-}}\right), 1.97\left(\mathrm{~m}, 2 \mathrm{H},-\mathrm{NC}_{5} \mathrm{H}_{10} \mathrm{CO}_{2}-\right.$ ), $1.69\left(\mathrm{~s}, 6 \mathrm{H},-\mathrm{O} 2\left(\mathrm{CH}_{3}\right)_{2}\right), 1.63(\mathrm{~m}, 2 \mathrm{H}$, $\left.\mathrm{NC}_{5} \mathrm{H}_{10} \mathrm{CO}_{2}-\right), 1.40\left(\mathrm{~m}, 2 \mathrm{H},-\mathrm{NC}_{5} \mathrm{H}_{10} \mathrm{CO}_{2}-\right.$ ), $1.24\left(\mathrm{t}, \mathrm{J}=7.0 \mathrm{~Hz}, 3 \mathrm{H},-\mathrm{CH}_{2} \mathrm{CH}_{3}\right)$.

\section{1-(5-Carboxypentyl)-2-}

\section{methylbenzo[c,d]indolium inner salt (18)}

Yield 75\%, M.p: $285-286{ }^{\circ} \mathrm{C} ;{ }^{1} \mathrm{H}$ NMR $\left.\left(300 \mathrm{MHz}, \mathrm{DMSO}-d_{6}\right)\right) \delta 8.95(\mathrm{~m}, 2 \mathrm{H}, \mathrm{ArH})$, $8.53(\mathrm{~m}, 2 \mathrm{H}, \mathrm{ArH}), 8.10$ (m, $2 \mathrm{H}, \mathrm{ArH}), 6.5$ (s, $3 \mathrm{H},-\mathrm{CH} 3), 2.24$ (m, $2 \mathrm{H}), 4.69$ (m, $2 \mathrm{H}$, $\mathrm{NC}_{5} \mathrm{H}_{10} \mathrm{CO}_{2^{-}}$), $1.98\left(\mathrm{~m}, 2 \mathrm{H},-\mathrm{NC}_{5} \mathrm{H}_{10} \mathrm{CO}_{2}\right.$ ), $1.60\left(\mathrm{~m}, 4 \mathrm{H},-\mathrm{NC}_{5} \mathrm{H}_{10} \mathrm{CO}_{2^{-}}\right) ;{ }^{13} \mathrm{C}$ NMR $(100$ MHz, DMSO- $\left.d_{6}\right) \delta 14.46,24.72,26.81,27.10$, $29.10,34.18,49.32,60.55,81.83,102.93$, $113.50,124.80,126.11,128.35,129.34$, $130.18,132.24,133.86,135.03,140.86$, 163.46, 166.32, 173.50. High-resolution ms (maldi, negative ion mode): calcd. for $\mathrm{C}_{18} \mathrm{H}_{20} \mathrm{NO}_{2} \quad\left(\mathrm{M}^{-}\right), \mathrm{m} / \mathrm{z} 282.15$; found $\mathrm{m} / \mathrm{z}$ 282.10 .

\section{Cyanine dye 21}

Yield $18 \% ; \lambda_{\max }(857 \mathrm{~nm}, \mathrm{MeOH})$; M.p: > $300{ }^{\circ} \mathrm{C}{ }^{1} \mathrm{H}$ NMR ( $300 \mathrm{MHz}$, DMSO$\left.\left.d_{6}\right)\right) 8.77(\mathrm{~m}, 2 \mathrm{H}, \mathrm{ArH}), 8.97(\mathrm{~d}, \mathrm{~J}=8.0 \mathrm{~Hz}, 2$ $\mathrm{H}, \operatorname{ArH}), 7.96(\mathrm{~m}, 2 \mathrm{H}, \mathrm{ArH}), 8.28(\mathrm{~d}, \mathrm{~J}=8$ $\mathrm{Hz}, 2 \mathrm{H}, \mathrm{ArH}), 7.67(\mathrm{t}, \mathrm{J}=13.5 \mathrm{~Hz}, 2 \mathrm{H}$, $\mathrm{ArH}), 7.80(\mathrm{~d}, \mathrm{~J}=8.0 \mathrm{~Hz}, 2 \mathrm{H}, \mathrm{ArH}), 7.59$ ( d, $\mathrm{J}=8.0 \mathrm{~Hz}, 2 \mathrm{H},-\mathrm{CH}=\mathrm{CH}-\mathrm{CH}=\mathrm{CH}-\mathrm{CH}=)$, $7.18(\mathrm{~m}, 1 \mathrm{H},-\mathrm{CH}=\mathrm{CH}-\mathrm{CH}=\mathrm{CH}-\mathrm{CH}=), 6.92$ (d, J = $13.5 \mathrm{~Hz}, 2 \mathrm{H}$, -CH=CH-CH=CH-CH=), $4.33\left(\mathrm{t}, \mathrm{J}=7 \mathrm{~Hz}, 4 \mathrm{H},-\mathrm{NC}_{5} \mathrm{H}_{10} \mathrm{CO}_{2}\right.$ ), $4.00(\mathrm{q}$, $\left.\mathrm{J}=7 \mathrm{~Hz}, 4 \mathrm{H},-\mathrm{CH}_{2} \mathrm{CH}_{3}\right), 2.32(\mathrm{t}, \mathrm{J}=7 \mathrm{~Hz}, 4$ $\left.\mathrm{H},-\mathrm{NC}_{5} \mathrm{H}_{10} \mathrm{CO}_{2}-\right), 1.84\left(\mathrm{~m}, 4 \mathrm{H},-\mathrm{NC}_{5} \mathrm{H}_{10} \mathrm{CO}_{2}-\right.$ ), $1.63\left(\mathrm{~m}, 4 \mathrm{H},-\mathrm{NC}_{5} \mathrm{H}_{10} \mathrm{CO}_{2}-\right), 1.44(\mathrm{~m}, 4 \mathrm{H}$, $\mathrm{NC}_{5} \mathrm{H}_{10} \mathrm{CO}_{2^{-}}$), $1.16(\mathrm{t}, \mathrm{J}=7.0 \mathrm{~Hz}, 6 \mathrm{H}$, $\mathrm{CH}_{2} \mathrm{CH}_{3}$ ); High-resolution $\mathrm{ms}$ (maldi, negative ion mode): calcd. for $\mathrm{C}_{43} \mathrm{H}_{47} \mathrm{~N}_{2} \mathrm{O}_{4}$ (M), $\mathrm{m} / \mathrm{z} 655.35$; found $\mathrm{m} / \mathrm{z} 655.42$.

\section{Cyanine dye 22}

Yield 13\%; $\lambda_{\max }(969 \mathrm{~nm}, \mathrm{MeOH})$; M.p: > $300{ }^{\circ} \mathrm{C}{ }^{1} \mathrm{H}$ NMR ( $300 \mathrm{MHz}$, DMSO$\left.\left.d_{6}\right)\right) 8.90(\mathrm{~d}, \mathrm{~J}=8.0 \mathrm{~Hz}, 2 \mathrm{H}, \mathrm{ArH}), 8.75(\mathrm{~m}, 2$ $\mathrm{H}, \operatorname{ArH}), 8.62(\mathrm{~m}, 2 \mathrm{H}, \operatorname{ArH}), 8.28(\mathrm{~d}, \mathrm{~J}=8$
$\mathrm{Hz}, 2 \mathrm{H}, \mathrm{ArH}), 7.92(\mathrm{~m}, 2 \mathrm{H}, \mathrm{ArH}), 7.78$ (d, J $=8.0 \mathrm{~Hz}, 2 \mathrm{H}, \mathrm{ArH}), 7.65(\mathrm{t}, \mathrm{J}=13.5 \mathrm{~Hz}, 2 \mathrm{H}$, $-\mathrm{CH}=\mathrm{CH}-\mathrm{CH}=\mathrm{CH}-\mathrm{CH}=\mathrm{CH}-\mathrm{CH}=), 7.60(\mathrm{~d}, \mathrm{~J}$ $=8.0 \mathrm{~Hz}, 2 \mathrm{H},-\mathrm{CH}=\mathrm{CH}-\mathrm{CH}=\mathrm{CH}-\mathrm{CH}=\mathrm{CH}-$ $\mathrm{CH}=), \quad 7.20(\mathrm{~m}, 1 \mathrm{H}, \quad-\mathrm{CH}=\mathrm{CH}-\mathrm{CH}=\mathrm{CH}-$ $\mathrm{CH}=\mathrm{CH}-\mathrm{CH}=), 6.90(\mathrm{~d}, \mathrm{~J}=13.5 \mathrm{~Hz}, 2 \mathrm{H}$, $\mathrm{CH}=\mathrm{CH}-\mathrm{CH}=\mathrm{CH}-\mathrm{CH}=\mathrm{CH}-\mathrm{CH}=), 4.35(\mathrm{t}, \mathrm{J}=$ $\left.7 \mathrm{~Hz}, 4 \mathrm{H},-\mathrm{NC}_{5} \mathrm{H}_{10} \mathrm{CO}_{2^{-}}\right), 4.00(\mathrm{q}, \mathrm{J}=7 \mathrm{~Hz}, 4$ $\left.\mathrm{H},-\mathrm{CH}_{2} \mathrm{CH}_{3}\right), 2.34(\mathrm{t}, \mathrm{J}=7 \mathrm{~Hz}, 4 \mathrm{H}$, $\left.\mathrm{NC}_{5} \mathrm{H}_{10} \mathrm{CO}_{2^{-}}\right), 1.82\left(\mathrm{~m}, 4 \mathrm{H},-\mathrm{NC}_{5} \mathrm{H}_{10} \mathrm{CO}_{2^{-}}\right)$, $1.62\left(\mathrm{~m}, 4 \mathrm{H},-\mathrm{NC}_{5} \mathrm{H}_{10} \mathrm{CO}_{2}-\right), 1.40(\mathrm{~m}, 4 \mathrm{H}$, $\mathrm{NC}_{5} \mathrm{H}_{10} \mathrm{CO}_{2}$ ), 1.15 (t, J = 7.0 Hz, $6 \mathrm{H}$, $\mathrm{CH}_{2} \mathrm{CH}_{3}$ ); High-resolution ms (maldi, negative ion mode): calcd. for $\mathrm{C}_{45} \mathrm{H}_{49} \mathrm{~N}_{2} \mathrm{O}_{4}$ $\left(\mathrm{M}^{-}\right), \mathrm{m} / \mathrm{z}$ 681.37; found $\mathrm{m} / \mathrm{z} 681.40$.

\section{Cyanine dye 25}

Yield $10 \% ; \lambda_{\max }(1011 \mathrm{~nm}, \mathrm{MeOH})$ M.p: > $300{ }^{\circ} \mathrm{C}{ }^{1} \mathrm{H}$ NMR ( $300 \mathrm{MHz}$, DMSO$\left.\left.d_{6}\right)\right) 8.32(\mathrm{~d}, \mathrm{~J}=7.5 \mathrm{~Hz}, 2 \mathrm{H}, \mathrm{ArH}), 7.87(\mathrm{~d}, \mathrm{~J}$ $=8 \mathrm{~Hz}, 2 \mathrm{H}, \operatorname{ArH}), 7.85(\mathrm{~d}, \mathrm{~J}=13.5 \mathrm{~Hz}, 2 \mathrm{H}$, ArH), $7.75(\mathrm{t}, \mathrm{J}=8.0 \mathrm{~Hz}, 2 \mathrm{H}, \mathrm{ArH}), 7.51$ ( $\mathrm{t}, \mathrm{J}$ $=8.0 \mathrm{~Hz}, 2 \mathrm{H}, \mathrm{ArH}), 7.40(\mathrm{~d}, \mathrm{~J}=8.0 \mathrm{~Hz}, 2 \mathrm{H}$, $\mathrm{ArH}), 7.03(\mathrm{~d}, \mathrm{~J}=7.5 \mathrm{~Hz}, 2 \mathrm{H},-\mathrm{CH}=\mathrm{CH}-$ chlorocycloxene), $6.21(\mathrm{~d}, \mathrm{~J}=13.5 \mathrm{~Hz}, 2 \mathrm{H}$, $\mathrm{CH}=\mathrm{CH}$-chlorocycloxene), $3.82(\mathrm{q}, \mathrm{J}=7 \mathrm{~Hz}$, $\left.4 \mathrm{H},-\mathrm{CH}_{2} \mathrm{CH}_{3}\right), 4.04\left(\mathrm{~m}, 8 \mathrm{H},-\mathrm{NC}_{5} \mathrm{H}_{10} \mathrm{CO}_{2}-\right.$ ), 2.78 (m, $2 \mathrm{H}$, -Clcyclohen), 2.27 (m, $4 \mathrm{H}$, Clcyclohene), $1.82\left(\mathrm{~m}, 4 \mathrm{H},-\mathrm{NC}_{5} \mathrm{H}_{10} \mathrm{CO}_{2}-\right)$, $1.63\left(\mathrm{~m}, 4 \mathrm{H},-\mathrm{NC}_{5} \mathrm{H}_{10} \mathrm{CO}_{2}-\right), 1.45(\mathrm{~m}, 4 \mathrm{H}$, $\left.\mathrm{NC}_{5} \mathrm{H}_{10} \mathrm{CO}_{2}-\right), 1.36\left(\mathrm{~m}, 6 \mathrm{H},-\mathrm{CH}_{2} \mathrm{CH}_{3}\right)$; Highresolution $\mathrm{ms}$ (maldi, negative ion mode): calcd. for $\mathrm{C}_{48} \mathrm{H}_{52} \mathrm{ClN}_{2} \mathrm{O}_{4}\left(\mathrm{M}^{-}\right), \mathrm{m} / \mathrm{z}$ 755.36; found $\mathrm{m} / \mathrm{z} 755.08$.

\section{DISCUSSION}

$\mathrm{N}$-alkylbenzo[c,d]indolium salts were used as starting compounds to prepare the squaraine dyes. The synthetic route to the starting compounds $\mathbf{1 0}$ and $\mathbf{1 1}$ involved five steps starting with $\mathbf{1}$ (Scheme 1) as described in literature (Strekowski et al., 2007).

We found that it was not necessary to purify the intermediate compounds $\mathbf{6}$ and $\mathbf{7}$ as the synthesis of $\mathbf{8}$ and $\mathbf{9}$ proceeded in good yield with the crude substrates. The third step of the synthesis of $\mathbf{1 1}$ did not go forward using methyl iodide reagent; we obtained a mixture of products including the substrate $\mathbf{5}$, the 
desired product and some side products. This unsuccessful reaction was probably due to the presence of the sulfonated group in the alkylated chain. Therefore, dimethylsulfate was used for this step and the reaction went forward to completion. A sticky oil product was obtained and used without purification for the next step.

The synthesis of the symmetrical squaraine dyes 12 and 13 was showed in Scheme 2. A mixture of a Nalkylbenzo[c,d]indolium salt containing an activated methyl group $\mathbf{1 0}$ or $\mathbf{1 1}$ (2 equiv) and 3,4-dihydroxy1,2 dioxocyclobut-3-ene (1 equiv) was heated under reflux in $n$ $\mathrm{BuOH} /$ Toluene $(7: 3)$ without using any catalyst (Schemes 2 and 3). Since squaraine dyes in general are brightly colored compounds with absorption in the visible or near infrared region, depending on the chromophore, we monitored the progress of the reaction by spectroscopy absorption in addition to the thin layer chromatography. No absorption band was observed during the initial stages of the reaction. However, an absorption band appeared after 1 hour, this band increased in intensity with reaction time. Isolation and purification could be achieved in the usual manner: silica gel column chromatography followed by recrystallization.

As described in literature, the synthetic route of 1-(5-carboxypentyl)-2methylbenzo[c,d]indolium inner salt $\mathbf{1 8}$ starting with $\mathbf{1}$ involves the intermediary of a derivative 16 (Lee et al., 2008; Shindy, 2015). Compound 16 was then alkylated with ethyl6-bromohexanoate and the resultant $\mathrm{N}$-(5ethylcarbonyl) pentyl derivative $\mathbf{1 7}$, after purification, was transformed to the desired iodide salt $\mathbf{1 8}$ by treatment with a mixture of acetic acid, hydrochloric acid, and potassium iodide. The substrate $\mathbf{1 8}$ was treated respectively with malonaldehyde dianilido hydrochloride, glutaconaldehyde dianilido hydrochloride and pentamethium salt $\mathbf{2 3}$ (a Vilsmeier-Haack reagent, obtained by formylation of cyclohexanone via the system $\mathrm{POCl}_{3} / \mathrm{CH}_{2} \mathrm{Cl}_{2} \mathrm{DMF} / \mathrm{PhNH}{ }_{3} \mathrm{Cl}$ ) (Strekowski et al., 2004) in acetic anhydride in the presence of sodium acetate as a base catalyst to furnish respectively the intermediary dyes 19, 20 and 24. TLC analysis showed a lot of byproducts. Attempts to purify these dyes by either flash column chromatography or crystallization were unsuccessful, probably this was due to carboxylic group. To overcome our goal, the crude products 19,20 and 24 were esterified by reacting with ethyl iodide in dimethylformamide in the presence of potassium carbonate as a base catalyst to give respectively crude ester-dye products $\mathbf{2 1}, \mathbf{2 2}$ and $\mathbf{2 5}$. The reaction progress was monitored by visible/near infrared spectroscopy for aliquots diluted with methanol until absorption of the intermediary starting cyanine disappeared. These cyanine dyes were purified respectively by colunmn chromatograpy and chromatotron using $\mathrm{CH}_{2} \mathrm{Cl}_{2} / \mathrm{MeOH} \quad(9: 1)$ as an eluent. The absorption maxima $\left(\lambda_{\max }\right)$ for the synthesized dyes were measured in methanol. These dyes were characterized by ${ }^{1} \mathrm{H}$ NMR, UV-vis spectra, MALDI mass spectrometry and Elemental analyses. Among all dyes, the heptamethine cyanine dye derivatives $22\left(\lambda_{\max }\right.$ $=969 \mathrm{~nm})$ and $25\left(\lambda_{\max }=1011 \mathrm{~nm}\right)$ showed high absorption; this was due to the increase number of vinylene groups compared to the squaraine dyes 12, 13 and the pentamethine cyanine dye $\mathbf{2 1}$ having their absorption in the range of 850 to $890 \mathrm{~nm}$. Interestingly, the three newly prepared near-infrared cyanine dyes containing functionalized $\mathrm{N}$-substituents 21, 22 and 25 could be used as intermediary products to prepare other cyanine dyes by transforming the ester moiety. In addition to this functionality, the chloro-substituted heptamethine cyanine dye $\mathbf{2 5}$ can readily undergo nucleophilic substitution at the meso position via a $\mathrm{SNR}_{1}$ mechanistic pathway to replace the chlorine atom with more versatile functionalities (Hammer et al., 2002; Lee et al., 2006).

\section{Conclusion}

Five new symmetrical dyes absorbing in the near- infrared region have been prepared by condensation of N- 
alkylbenzo[c,d]indolium salts having an active 2-methyl group with either squaric acid or imine. The three near-infrared cyanine dyes 21, 22 and 25 containing functionalized $\mathrm{N}$ substituents (ester group) could be further modified or covalently attached to target analytes. In addition, the chloro-substituted heptamethine cyanine dye $\mathbf{2 5}$ which has the highest absorption $\left(\lambda_{\max }=1011 \mathrm{~nm}\right)$ can readily undergo nucleophilic substitution at the meso position via a $\mathrm{SNR}_{1}$ mechanistic pathway to replace the chlorine atom with more versatile functionalities. Further, these molecules will be tested to establish their activities on nucleic acids and proteins.

\section{COMPETING INTERESTS}

The authors declare that they have no competing interests.

\section{AUTHORS' CONTRIBUTIONS}

VMS carried out all synthesized compounds and wrote the manuscript. TAO has helped in the spectroscopic interpretations, made some inputs in the manuscript and validated the final version of the manuscript. SJA has contributed to the manuscript writing and interpretation of some data. AA has supervised all works related to manuscript.

\section{REFERENCES}

Bouteiller C, Clavé G, Bernardin A, Chipon B, Massonneau M, Renard PY, Romieu A. 2007. Novel water-soluble nearinfrared cyanine dyes: synthesis, spectral properties, and use in the preparation of internally quenched fluorescent probes. Bioconjugate Chem., 18 (4): 1303-1317.

Doi: $10.1021 / \mathrm{bc} 0700281$.

Hammer RP, Owens CV, Hwang SH, Sayes CM, Soper SS. 2002. Asymmetrical, water-soluble phthalocyanine dyes for covalent labeling of oligonucleotides. Bioconjugate Chem., 13 (6): 1244-1252. Doi: 10.1021/bc0155869.
Hyun H, Owens EA, Wada H, Levitz A, Park G, Park MH, Frangioni JV, Henary M, Choi HS. 2015. Cartilage-specific nearinfrared fluorophores for biomedical imaging. Angew. Chem. Int. Ed. Engl., 54(30): 8648-8652. Doi: 10.1002/anie.201502287.

Hyun H, Park MH, Owens EA, Wada H, Henary M, Henricus JMH, Alexander LV, Frangioni JV, Choi HS. 2015. Structure-inherent targeting of nearinfrared fluorophores for parathyroid and thyroid gland imaging. Nat. Med., 21: 192-197. Doi: 10.1038/nm.3728.

Lee H, Berezin MY, Henary M, Strekowski L, Achilefu SJ. 2008. Fluorescence lifetime properties of near-infrared cyanine dyes in relation to their structures. Photochem. Photobiol. A. Chem., 200(2-3): 438-444. Doi: 10.1016/j.jphotochem.2008.09.008.

Lee H, Mason JC, Achilefu G J. 2006. Heptamethine cyanine dyes with a robust $\mathrm{C}-\mathrm{C}$ bond at the central position of the chromophore. J. Org. Chem., 71(20): 7862-7865._Doi: 10.1021/jo061284u.

Liu Y, Zhang Z, Chen X, Xu S, Cao S. 2016. Near-infrared absorbing dyes at $1064 \mathrm{~nm}$ : soluble dithiolene nickel complexes with alkylated electrondonating groups as peripheral substituents. Dyes Pigm., 128: 179-189. https://doi.org/10.1016/j.dyepig.2016.01. 026.

Njiojob CN, Owens EA, Narayana L, Hyun H, Choi H, Henary M. 2015. Tailored nearinfrared contrast agents for image guided surgery. J. Med. Chem., 58 (6): 28452854.

Doi: 10.1021/acs.jmedchem.5b00253.

Pisoni DS, Todeschini L, Borges ACA, Petzhold CL, Rodembusch FS, Campo LF. 2014. Symmetrical and asymmetrical cyanine dyes. Synthesis, spectral properties, and BSA association study. J. Org. Chem., 79 (12): 55115120. Doi: $10.1021 /$ jo500657s. 
Rauch T, Böberl M, Tedde SF, Fürst J, Kovalenko MV, Hesser G, Lemmer U, Heiss W, Hayden O. 2009. Near infrared imaging with quantum-dot- sensitized organic photodiode. Nat. Photonics, 3: 332-336. Doi: 10.1038/nphoton.2009.72.

Shindy HA. 2015. Synthesis of different classes of benz (naphth) / five membered heterocyclic cyanine dyes. Rev. Roum. Chim., 60 (1): 5-13.

Strekowski L, Lee H, Mason JC, Say M, Patonay GJ. 2007. Stability in solution of indolium heptamethine cyanines and related $\mathrm{pH}$-sensitive systems. Heterocyclic Chem., 44: 475-482. Doi: 10.1002/jhet.5570440233.

Strekowski L, Mason JC, Lee H, Say M, Patonay GJ. 2004. Water-soluble pH- sensitive 2,6-bis(substituted ethylidene)cyclohexanone/hydroxyl cyanine dyes that absorbe in the near-infrared region. Heterocycl. Chem., 41 (2): 227-232. https://doi.org/10.1002/jhet.5570410213. Volkva KD, Kovalska VB, Tataret AL, Patsenker LD, Kryvorotenko DV, Yarmoluk SM. 2007. Spectroscopic study of squaraines as protein-sensitive fluorescent dyes. Dyes Pigm., 72 (3): 285-292.

Doi: 10.1016/j.dyepig.2005.09.007.

Zhang H, Jenatsch S, Jonghe JD, Nuesch F, Steim R, Veron AC, Hany R. 2015. Transparent organic photodetector using a near-infrared absorbing cyanine dye. Sci. Rep., 5: 9439-9445. Doi: 10.1038/srep09439. 